\title{
20(S)-Protopanaxadiol Induces Human Breast Cancer MCF-7 Apoptosis through a Caspase-Mediated Pathway
}

\author{
Hong Zhang ${ }^{\star}$, Hua-Li Xu ${ }^{\star}$, Wen-wen Fu, Ying Xin, Mao-Wei Li, Shuai-Jun \\ Wang, Xiao-Feng Yu*, Da-Yun Sui*
}

\begin{abstract}
20(S)-Protopanaxadiol (PPD), a ginsenoside isolated from Pananx quinquefolium L., has been shown to inhibit growth and proliferation in several cancer cell lines. The aim of this study was to evaluate its anticancer activity in human breast cancer cells. MCF-7 cells were incubated with different concentrations of 20(S)-PPD and cytotoxicity was evaluated by MTT assay. Occurrence of apoptosis was detected by DAPI and Annexin V-FITC/PI double staining. Mitochondrial membrane potential was measured with Rhodamine 123. The Bcl-2 and Bax expression were determined by Western blot analysis. Caspase activity was measured by colorimetric assay. 20(S)-PPD dose-dependently inhibited cell proliferation in MCF-7 cells, with an IC F0 $_{50}$ value of $33.3 \mu \mathrm{M}$ at 24h. MCF-7 cells treated with 20(S)-PPD presented typical apoptosis, as observed by morphological analysis in cell stained with DAPI. The percentages of annexin V-FITC positive cells were $8.92 \%, 17.8 \%, 24.5 \%$ and $30.5 \%$ in MCF-7 cells treated with $0,15,30$ and $60 \mu \mathrm{M}$ of $20(\mathrm{~S})$-PPD, respectively. Moreover, 20(S)-PPD could induce mitochondrial membrane potential loss, up-regulate Bax expression and down-regulate Bcl-2 expression. These events paralleled activation of caspase-9, -3 and PARP cleavage. Apoptosis induced by 20(S)-PPD was blocked by z-VAD-fmk, a pan-caspase inhibitor, suggesting induction of caspase-mediated apoptotic cell death. In conclusion, the 20(S)-PPD investigated is able to inhibit cell proliferation and to induce cancer cell death by a caspase-mediated apoptosis pathway.
\end{abstract}

Keywords: 20(S)-Protopanaxadiol - apoptosis - caspase - ginsenoside - MCF-7 breast cancer cells

Asian Pac J Cancer Prev, 15 (18), 7919-7923

\section{Introduction}

Cancer is one of the major diseases leading to death in the world (Ma et al., 2014). Breast cancer is the first leading cause of death among the women aged 20-59 years (Siegel et al., 2013; Zhu et al., 2013). It is estimated that 40, 000 women will die of breast cancer and 234, 000 new cases will be diagnosed each year (Colditz and Bohlke, 2014). The standard treatment for breast cancer included surgical therapy, chemotherapy, radiotherapy, hormonotherapy (Florescu et al., 2011). Chemotherapy or radiotherapy has harmful effect on both cancer cell and normal cells. So, novel drugs which are effective and safe for patient are urgently needed.

Nowadays, natural products are considered as a significant resource for potential drugs (Gao et al., 2013). Since the 1940s, over $60 \%$ of new small molecules of anticancer drugs were natural products or their derivatives (Newman and Cragg, 2012). Ginseng, the root of different Panax species, has been used as traditional herbal medicine in East Asian countries for thousands of years. The major pharmacological active ingredients of ginseng are ginsenosides which have many biological activities including antihyerlipidemic (Quan et al., 2012), antidiabetic effect (Attele et al., 2002), antioxidation (Zhang et al., 1996), immunostimulation (Shin et al., 2002), antistress (Wang and Lee, 2000) and anticancer (Lin et al., 2013). Ginsenosides are divided into the protopanaxadiol, protopananatiol and oleanolic acid ginsenosides according to their structure (Xu et al., 2010a; Wang et al., 2012).

20 (S)-Protopanaxadiol (PPD) is an active ginseng metabolite, which is the final form of protopanaxadiol saponins metabolized by human intestinal microflora (Figure 1) (Xie et al., 2009). It was reported that 20 (S)PPD showed anticancer effect in experimental animals and cultured cells though caspase-dependent and caspaseindependent pathway (Oh and Lee, 2004; Liu et al., 2007; Yu et al., 2007; Wang et al., 2008). At present, 20 (S)-PPD has been developed into a Chinese medicine, named "Yijinsheng Capsule", to assist radiotherapy and chemotherapy, currently in clinical stage III. Our 


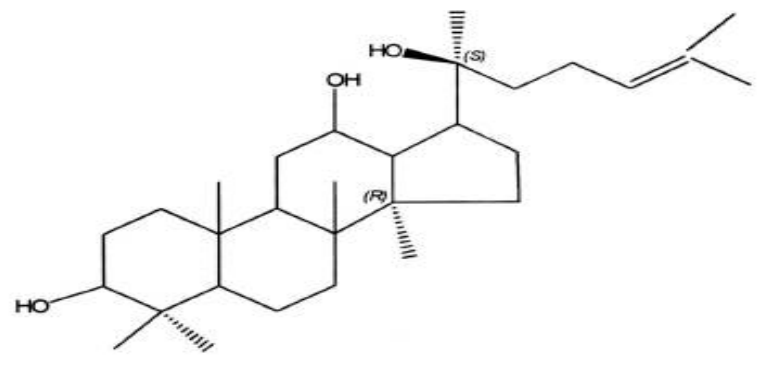

Figure 1. Chemical Structure of 20(S)-Protopanaxadiol (PPD)

previous study demonstrated that 20 (S)-PPD could induce apoptosis in A549 cell via the mitochondrial pathway and inhibited the phosphorylation of AKT (Zhang et al., 2013b). However, the mechanism of cytotoxicity of 20 (S)-PPD has not been investigated. Therefore, in the present study, we investigated the cytotoxic mechanism of 20 (S)-PPD in human breast cancer MCF-7 cells.

\section{Materials and Methods}

\section{Chemicals}

20 (S)-Protopanaxadiol (PPD) was provided by Hainan Asian Pharmaceutical Co. Led., (China). The purity of 20 (S)-PPD used in experiments was $>95 \%$ detected by HPLC. Antibodies against pro-caspase-3/-9, PARP, Bcl-2, Bax were purchased from Cell Signal Technology (Beverly, MA, USA). Antibody against $\beta$-actin was obtained from Tianjing Jingmai.Z-VAD-fmk was ordered from Santa Cruz Biotechology (Dallas, TX, USA). BCA protein assay reagent kit, DAPI staining kit, caspase activity assay kit and Rhodamine 123 were purchased from Beyotime Institue of Biotechnology (Jiangsu, China). Annexin V-FITC apoptosis detection kit was obtained from Tianjin Sungene Biotech Co. Ltd (Tianjin, China). MTT and all other reagents were purchased from SigmaAdrich Co. (St. Louis, MO, USA).

\section{Cell culture and treatment}

Human breast cancer MCF-7 cells were obtained from Shanghai Institute of Cell Biology, Chinese Academic of Science (Shanghai, China). MCF-7 cells were cultured in RPMI-1640 medium (Hyclone, Logan, UT, USA) supplemented with $10 \%$ heat-inactivated $\left(56^{\circ} \mathrm{C}, 30 \mathrm{~min}\right)$ fetal calf serum (GIBCO, Grand Island, NY, USA) under standard cultured conditions $\left(37^{\circ} \mathrm{C}, 95 \%\right.$ humidified air and $5 \% \mathrm{CO}_{2}$ ). $20(\mathrm{~S})$-PPD dissolved in DMSO and was added to the culture media to the final concentrations.

\section{MTT assay}

Cell viability was measured by MTT assay as described previous (Xu et al., 2010b). Briefly, the MCF-7 cells were seeded into 96-well plates, and treated with different concentration of 20 (S)-PPD (the final concentration of was $5,10,20,40,80 \mu \mathrm{M})$. After incubation for the 20h, $10 \mu \mathrm{l}$ of MTT (Sigma, $5 \mathrm{mg} / \mathrm{ml}$ in PBS) solution was added to each well and plates were then incubated another for $4 \mathrm{~h}$. Then $100 \mu \mathrm{l}$ of dimethyl sulfoxide (DMSO) was added to each well, and the plates were shaken for $10 \mathrm{~min}$. The absorbance was read at $570 \mathrm{~nm}$ with a microplate reader (SpectraMax Plus384, Molecular Devices, USA). Percentage of survival was calculated as a fraction of the negative control. The half-maximal inhibitory concentration $\left(\mathrm{IC}_{50}\right)$ was calculated from the dose-response curve by original 6.0 software.

\section{DAPI staining}

The DAPI staining was performed as previously described (Matin et al., 2014). Briefly, MCF-7 cells were seeded on coverslips with different concentrations of 20 (S)-PPD for $24 \mathrm{~h}$. The coverslips were washed twice with PBS, fixed in $4 \%$ paraformaldehyde, permeabilized with $0.1 \%$ Triton X-100 and stained with $2 \mu \mathrm{g} / \mathrm{ml}$ DAPI for $10 \mathrm{~min}$. Finally, the cells were observed under the fluorescence microscope (Nikon TE-2000U, Nikon Corporation, Tokyo, Japan).

\section{Annexin V-FITC/PI assay}

To quantify the apoptosis induced by 20 (S)-PPD in MCF-7 cells, Annexin V-FITC/PI staining was done by flow cytometry as previously described (Xu et al., 2010b). Briefly, after 20 (S)-PPD treatment, MCF-7 cells were collected and washed twice in ice cold PBS and resuspended in $300 \mu \mathrm{l}$ of binding buffer containing $1 \mu \mathrm{g} /$ $\mathrm{ml} \mathrm{PI}$ and $0.05 \mu \mathrm{g} / \mathrm{ml}$ Annexin V-FITC. The samples were incubated for $15 \mathrm{~min}$ at room temperature in dark and were analyzed by flow cytometry.

\section{Mitochondrial membrane potential}

Mitochondrial membrane potential was measured by using the mitochondrial membrane potential sensitive cationic dye Rhodamine 123 as previously described (Bomfim et al., 2013). Briefly, MCF-7 cells treated with different concentration of 20 (S)-PPD for $24 \mathrm{~h}$ and incubated with Rhodamine 123 at $37^{\circ} \mathrm{C}$ for $30 \mathrm{~min}$. Flourescence intensities were analyzed by flow cytometric analysis.

\section{Caspase activity assay}

The activities of caspase-3, -9 were measured by colorimetric protease assay according to the manufacture's protocol. Briefly, cell lysates were prepared in cell lysis buffer for $15 \mathrm{~min}$ on ice and centrifuged at $18,000 \times \mathrm{g}$ for $10 \mathrm{~min}$ at $4^{\circ} \mathrm{C}$. The supernatants were collected and total protein was quantified by Bradford method. Protein lysate was mixed with reaction buffer (Ac-DEVD-pNA for caspase-3, Ac-LEHD-pNA for caspase -9) and incubated at $37^{\circ} \mathrm{C}$ for $2 \mathrm{~h}$ in the dark. Developed color was measured at $405 \mathrm{~nm}$ in microplate reader. Results are represented as the percentage of change of activity compared to the control.

\section{Western blot analysis}

Western blot was performed for detection of procaspase-9, -3 PARP, Bax and Bcl-2 proteins. After treatment with different concentration of 20 (S)-PPD, the MCF-7 cells were harvested and lysed in RIPA buffer for $30 \mathrm{~min}$ on ice. The protein concentration was determined using the BCA protein assay kit. The cell extract $(20 \mu \mathrm{g})$ were loaded onto $12 \%$ polyacrylamide-SDS gel. After electrophoresis, the gel was blotted onto a PVDF 


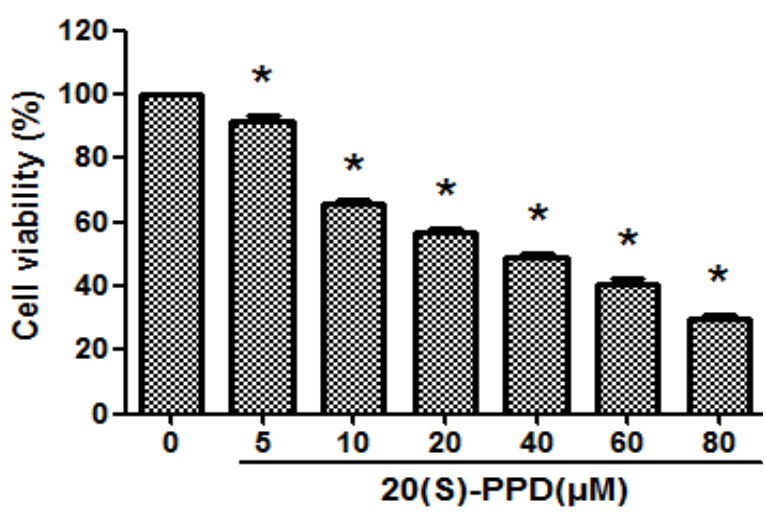

Figure 2. Effect of 20(S)-Protopanaxadiol on Cytotoxicity of MCF-7 Cells after 24h Exposure. MCF-7 cells were seeded in 96-well plates and incubated with different concentration of 20(S)-PPD for 24h. Cell viability was determined by MTT assay. Values are means \pm SD of three experiments. ${ }^{*} p<0.05$ compared to $0 \mu \mathrm{M}$

A
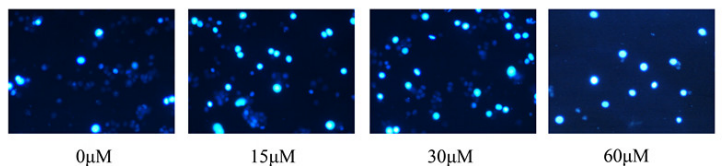

B

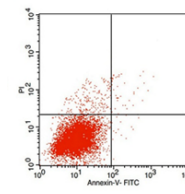

$0 \mu \mathrm{M}$

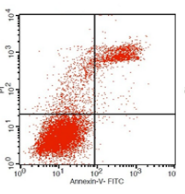

$15 \mu \mathrm{M}$

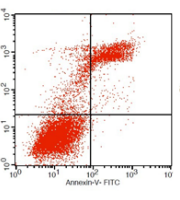

$30 \mu \mathrm{M}$
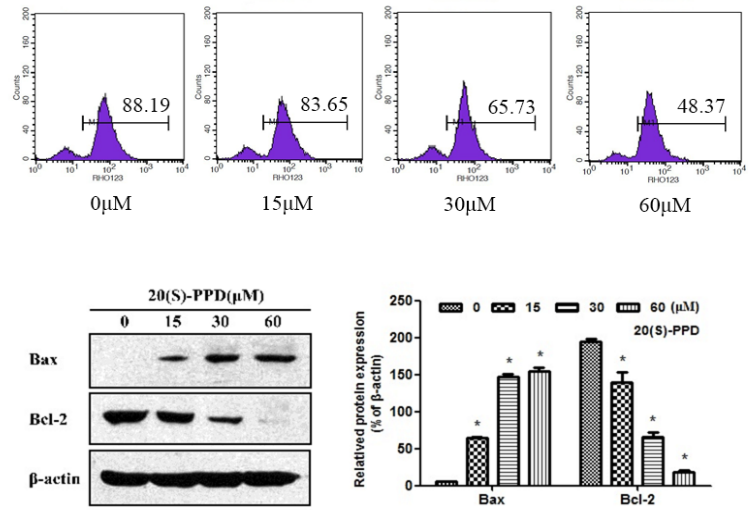

Figure 4. Effect of 20(S)-PPD on Mitochondrial Membrane Potential (MMP) and Bcl-2 Family Proteins. (A) MCF-7 cells were incubated with different concentrations of 20(S)-PPD $(15,30$ and $60 \mu \mathrm{M})$ for 24 $\mathrm{h}$ and incubated with Rhodamine 123 dye for another $30 \mathrm{~min}$. Fluorescence emission was measured by flow cytometry. (B) MCF-7 cells were treated with different concentrations of 20(S)-PPD $(15,30$ and $60 \mu \mathrm{M})$ for $24 \mathrm{~h}$ and Bcl-2 and Bax were determined by Western blot. All values were expressed as mean \pm SD. ${ }^{*} p<0.05,{ }^{* *} p<0.01$ compared to control

In an attempt to elucidate whether the loss in MCF-7 cell viability induced by 20 (S)-PPD was associated with apoptosis, the morphology changes were identified by DAPI staining. As shown in Figure 3A, DAPI staining showed relatively many apoptotic bodies containing nuclear fragments in MCF-7 cells treated with 20 (S)$\mathrm{PPD}$, but few were observed in control group. These data confirmed that 20 (S)-PPD induced apoptosis in MCF-7 cells. Furthermore, we also detected the apoptotic rate by Annexin V-FITC/PI staining. As shown in Figure 3B, the percentage of Annexin V-FITC positive cells increased with 20 (S)-PPD treatment. The apoptotic rate was $8.92 \%$, $17.83 \%, 24.52 \%$ and $30.51 \%$ in MCF-7 cells treated with $0,15,30$ and $60 \mu \mathrm{M}$ of $20(\mathrm{~S})$-PPD, respectively.

To further investigate the role of mitochondria in the apoptosis induced by 20 (S)-PPD, a flow cytometric analysis was performed. As shown in Figure 4A, treatment with $0,5,30$ and $60 \mu \mathrm{M} 20$ (S)-PPD for 24h, the MMP was decreased from $85.78 \%$ to $81.41 \%, 64.37 \%$ and $43.79 \%$, respectively. With 20 (S)-PPD treatment, Bcl-2 protein level was down-regulated while Bax expression was upregulated (Figure 4B).

As shown in Figure 5A, the caspase-3 and caspase-9 activities were markedly increased after treatment with different concentration of 20 (S)-PPD for $24 \mathrm{~h}$. Additionally, Figure 5B showed that z-VAD-fmk, a pan-caspase inhibitor, markedly attenuated the apoptosis induced by 20 (S)-PPD. As shown in Figure 5C, the cleavage of precursor of caspase- 9 and capase-3 were noted with the presence of 20 (S)-PPD in a dose-dependent manner. PARP is a substrate for caspase-3. 20 (S)-PPD treatment caused cleavage of PARP, $116 \mathrm{kDa}$ to $89 \mathrm{kDa}$ fragment (Figure 5C). This corresponded with the activation of caspase-3.These suggested that 20 (S)-PPD induced apoptosis engages caspase-dependent signaling 

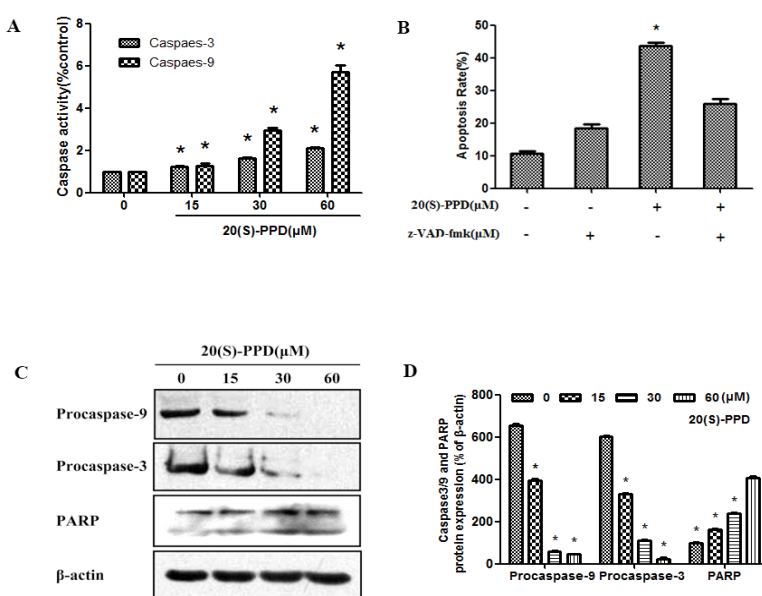

D

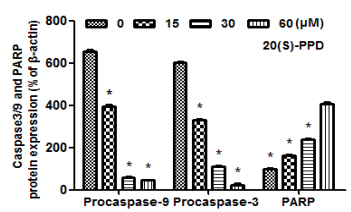

Figure 5. 20(S)-PPD Induced Apoptosis in MCF-7

Cells Via Mitochondria Pathway. (A) MCF-7 cells were incubated with different concentrations of 20(S)-PPD for 24h and caspase-9/-3 activities were assessed by the substrate AcLEHD-pNA and Ac-DEVD-pNA, respectively. (B) MCF-7 cells were incubated for $1 \mathrm{~h}$ at $37^{\circ} \mathrm{C}$ in the absence or presence of z-VAD-fmk, pan-caspase inhibitor at a concentration of $20 \mu \mathrm{M}$, prior to exposure to $60 \mu \mathrm{M}$ of $20(\mathrm{~S})$-PPD. Twenty-four hours later, the apoptosis was examined.(C),(D) Proteins expression of caspases-9/-3 and PRAP were determined by Western blot. All values were expressed as mean \pm SD. ${ }^{*} p<0.05,{ }^{*} * p<0.01$ compared to control

cascades. Taken together, our data suggest that 20 (S)PPD induced apoptosis via caspase-mediated pathway in MCF-7 cells.

\section{Discussion}

Cancer has become an increasing public health issue for its high rates of morbidity and mortality. In the current study, the anticancer activity of 20 (S)-PPD was investigated. The experimental results showed that 20 (S)-PPD exhibited cytotoxicity to human breast cancer MCF-7 cells, with an $\mathrm{IC}_{50}$ of $33.33 \mu \mathrm{M}$ (Figure 2). Apoptosis assay showed that apoptotic cells induced by 20 (S)-PPD exhibited cellular alterations, chromatin condensation by DAPI staining (Figure 3A). Annexin V-FITC/PI double-staining assay further confirmed the results of DAPI staining and the percentage of apoptosis increased in 20 (S)-PPD treatment (Figure 3B). All these results showed that $20(\mathrm{~S})-\mathrm{PPD}$ increased anticancer activity through apoptosis.

Apoptosis is a cellular suicidal mechanism that regulates normal physiological processes and plays a crucial role in both the development and maintenance of tissue homeostasis (Hail et al., 2006). The mitochondria play a pivotal role in apoptosis (Adams and Cory, 2007). Here, the changes of mitochondrial membrane potential were analyzed by the Rhodamine 123 dye, the apoptosis is mediated by a rapid dissipation of mitochondrial membrane potential with $24 \mathrm{~h}$ (Figure 4A). Bcl-2 family proteins are reported in regulation the function of mitochondria. Bcl-2 can stabilize mitochondria; in contrast, Bax increases the membrane permeability (Ly et al., 2003; Singh et al., 2013). It is also reported that an increased ratio of $\mathrm{Bax} / \mathrm{Bcl}-2$ triggered the apoptosis. In this study, we observed that 20 (S)-PPD induced up-regulation of Bax and down-regulation of Bcl-2 (Figure 4B). It was supposed that 20 (S)-PPD might regulate the mitochondria function through disruption of a balance between the Bcl-2 and Bax protein. These results of the current study were consistent with the previously studies in other cancer cell types including human lung adenocarcinoma cells (Zhang et al., 2013b), colon cancer cells (Zhang et al., 2013a) and prostate cancer cells (Chen et al., 2013).

The caspase proteases are believed to play a critical role in mediating apoptosis. Two different caspase pathways (extrinsic and intrinsic pathways) are involved in mediating the response. Both extrinsic and intrinsic pathways lead to activation executioner caspase (caspase-3). Caspase-3 activated by caspase-8 (extrinsic pathway) or caspase-9 (intrinsic pathway) induces cell shrinkage, nuclear condensation and DNA fragmentation (Zhou et al., 2013). Here, we showed that 20 (S)-PPD treatment activated caspase- 9 and caspase- 3 in a dosedependent manner (Figure 5A). Caspase-3 activation was dominant and reflected in the cleavage of PARP, a well-know substrate of caspase-3. During apoptosis, PARP was cleaved the precursor $116 \mathrm{kDa}$ to yield $85 \mathrm{kDa}$ fragments (Allen et al., 1997). As shown in Figure 5C, 20 (S)-PPD treatment caused the cleavage of PARP. This is corresponded with the activation of caspase-3 (Figure 5A). Moreover, the apoptosis induced by 20 (S)-PPD was markedly attenuated by z-VAD-fmk (Figure 5B), a pancaspase inhibitor. Taken together, our data suggest that 20 (S)-PPD induced apoptosis in MCF-7 cells through the activation of caspase cascades. However, further investigations were performed to highlight the apoptotic pathways involved in the apoptosis induced by 20 (S)-PPD in MCF-7 cells.

In conclusion, $20(\mathrm{~S})-\mathrm{PPD}$ decreased mitochondrial membrane potential, enhanced the expression of Bax, activated the casapase- 9 and caspase- 3 and, subsequently, induced apoptosis in human breast cancer MCF-7 cells. These results showed the potential benefits of 20 (S)-PPD for clinical practice.

\section{Acknowledgements}

This work was financially supported by the National Natural Science Foundation of China (Grant No. 81173602). The authors thank Xuejin Su for his assistance in flow cytometry data acquisition.

\section{References}

Adams JM, Cory S (2007). The Bcl-2 apoptotic switch in cancer development and therapy. Oncogene, 26, 1324-37.

Allen RT, Hunter WJ, 3rd, Agrawal DK (1997). Morphological and biochemical characterization and analysis of apoptosis. J Pharmacol Toxicol Methods, 37, 215-28.

Attele AS, Zhou YP, Xie JT, et al (2002). Antidiabetic effects of Panax ginseng berry extract and the identification of an effective component. Diabetes, 51, 1851-8.

Bomfim DS, Ferraz RP, Carvalho NC, et al (2013). Eudesmol isomers induce caspase-mediated apoptosis in human hepatocellular carcinoma HepG2 cells. Basic Clin Pharmacol Toxicol, 113, 300-6. 
Chen G, Yang X, Nong S, etal(2013). Two novel hydroperoxylated products of 20 (S)-protopanaxadiol produced by Mucor racemosus and their cytotoxic activities against human prostate cancer cells. Biotechnol Lett, 35, 439-43.

Colditz GA, Bohlke K (2014). Priorities for the primary prevention of breast cancer. CA Cancer J Clin, 64, 186-94.

Florescu A, Amir E, Bouganim N, et al (2011). Immune therapy for breast cancer in 2010-hype or hope? Curr Oncol, 18, 9-18.

Gao JL, Lv GY, He BC, et al (2013). Ginseng saponin metabolite 20 (S)-protopanaxadiol inhibits tumor growth by targeting multiple cancer signaling pathways. Oncol Rep, 30, 292-8.

Hail N, Jr., Carter BZ, Konopleva M, et al (2006). Apoptosis effector mechanisms: a requiem performed in different keys. Apoptosis, 11, 889-904.

Lin G, Yu X, Wang J, et al (2013). Beneficial effects of 20 (S)-protopanaxadiol on antitumor activity and toxicity of cyclophosphamide in tumor-bearing mice. Exp Ther Med, 5, 443-7.

Liu GY, Bu X, Yan H, et al (2007). 20S-protopanaxadiolinduced programmed cell death in glioma cells through caspase-dependent and -independent pathways. J Nat Prod, 70, 259-64.

Ly JD, Grubb DR, Lawen A (2003). The mitochondrial membrane potential (deltapsi (m)) in apoptosis; an update. Apoptosis, 8, 115-28.

Ma WD, Zou YP, Wang P, et al (2014). Chimaphilin induces apoptosis in human breast cancer MCF-7 cells through a ROS-mediated mitochondrial pathway. Food Chem Toxicol. 70, 1-8.

Matin MM, Nakhaeizadeh H, Bahrami AR, et al (2014). Ferutinin, an Apoptosis Inducing Terpenoid from Ferula ovina. Asian Pac J Cancer Prev, 15, 2123-8.

Newman DJ, Cragg GM (2012). Natural products as sources of new drugs over the 30 years from 1981 to 2010. J Nat Prod, 75, 311-35.

Oh SH, Lee BH (2004). A ginseng saponin metabolite-induced apoptosis in HepG2 cells involves a mitochondria-mediated pathway and its downstream caspase- 8 activation and Bid cleavage. Toxicol Appl Pharmacol, 194, 221-9.

Quan HY, Yuan HD, Jung MS, et al (2012). Ginsenoside Re lowers blood glucose and lipid levels via activation of AMPactivated protein kinase in HepG2 cells and high-fat diet fed mice. Int J Mol Med, 29, 73-80.

Shin JY, Song JY, Yun YS, et al (2002). Immunostimulating effects of acidic polysaccharides extract of Panax ginseng on macrophage function. Immunopharmacol Immunotoxicol, 24, 469-82.

Siegel R, Naishadham D, Jemal A (2013). Cancer statistics, 2013. CA Cancer J Clin, 63, 11-30.

Singh DV, Agarwal S, Singh P, et al (2013). Curcumin conjugates induce apoptosis via a mitochondrion dependent pathway in MCF-7 and MDA-MB-231 cell lines. Asian Pac J Cancer Prev, 14, 5797-804.

Wang CZ, Calway T, Yuan CS (2012). Herbal medicines as adjuvants for cancer therapeutics. Am J Chin Med, 40, 657-69.

Wang LC, Lee TF (2000). Effect of ginseng saponins on cold tolerance in young and elderly rats. Planta Med, 66, 144-7.

Wang W, Rayburn ER, Hao M, et al (2008). Experimental therapy of prostate cancer with novel natural product anti-cancer ginsenosides. Prostate, 68, 809-19.

Xie JT, Wang CZ, Zhang B, et al (2009). In vitro and in vivo anticancer effects of American ginseng berry: exploring representative compounds. Biol Pharm Bull, 32, 1552-8.

Xu C, Teng J, Chen W, et al (2010a). 20 (S)-protopanaxadiol, an active ginseng metabolite, exhibits strong antidepressant-like effects in animal tests. Prog Neuropsychopharmacol Biol Psychiatry, 34, 1402-11.

Xu HL, Yu XF, Qu SC, et al (2010b). Anti-proliferative effect of Juglone from Juglans mandshurica Maxim on human leukemia cell HL-60 by inducing apoptosis through the mitochondria-dependent pathway. Eur J Pharmacol, 645, 14-22.

Yu Y, Zhou Q, Hang Y, et al (2007). Antiestrogenic effect of 20S-protopanaxadiol and its synergy with tamoxifen on breast cancer cells. Cancer, 109, 2374-82.

Zhang D, Yasuda T, Yu Y, et al (1996). Ginseng extract scavenges hydroxyl radical and protects unsaturated fatty acids from decomposition caused by iron-mediated lipid peroxidation. Free Radic Biol Med, 20, 145-50.

Zhang R, Chung Y, Kim HS, et al (2013a). 20-O- (beta-Dglucopyranosyl)-20 (S)-protopanaxadiol induces apoptosis via induction of endoplasmic reticulum stress in human colon cancer cells. Oncol Rep, 29, 1365-70.

Zhang YL,Zhang R, Xu HL, et al (2013b). 20 (S)-protopanaxadiol triggers mitochondrial-mediated apoptosis in human lung adenocarcinoma A549 cells via inhibiting the PI3K/Akt signaling pathway. Am J Chin Med, 41, 1137-52.

Zhou J, Luo YH, Wang JR, et al (2013). Gambogenic acid induction of apoptosis in a breast cancer cell line. Asian Pac $J$ Cancer Prev, 14, 7601-5.

Zhu S, Yao F, Li WH, et al (2013). PKC?-dependent activation of the ubiquitin proteasome system is responsible for high glucose-induced human breast cancer MCF-7 cell proliferation, migration and invasion. Asian Pac J Cancer Prev, 14, 5687-92. 